\section{Multiple non-syndromic basal cell carcinoma with the chest as primary site and lung metas- tases: A rare case}

\author{
Konstantinos Roditis, ${ }^{1,2}$ \\ George Metaxas, ${ }^{3}$ Ourania Neofotistou, ${ }^{4}$ \\ Kleo Papaparaskeva, ${ }^{5}$ Aikaterini \\ Koutsoumbi, ${ }^{6}$ Konstantinos Louis ${ }^{2,7}$ \\ ${ }^{1}$ Department of Vascular Surgery, \\ Korgialenio-Benakio Hellenic Red Cross \\ Hospital, Athens; ' Junior Doctors' \\ Network-Hellas (JDN-Hellas), Athens; \\ ${ }^{3} 2^{\text {nd }}$ Surgical Department, Helena \\ Venizelou General and Maternity \\ District Hospital, Athens; ${ }^{4}$ Department \\ of Dermatology, Konstantopouleio Neas \\ Ionias-Patission General Hospital, Nea \\ Ionia, Athens; ${ }^{5}$ Department of \\ Pathology, Konstantopouleio Neas \\ Ionias-Patission General Hospital, Nea \\ Ionia, Athens; ${ }^{6}$ Department of \\ Pathology, Helena Venizelou General \\ and Maternity District Hospital, Athens; \\ ${ }^{7}$ Third Department of Obstetrics and \\ Gynecology, Attikon University General \\ Hospital, National and Kapodistrian \\ University of Athens, Medical School, \\ Athens, Greece
}

\section{Abstract}

Basal cell carcinoma (BCC) is the most commonly diagnosed cancer in humans, usually affecting elderly Caucasian men and skin regions mostly exposed to the sun, that rarely metastasizes. We report an unusual and aggressive case of multiple, non-syndromic metastatic BCC with an uncommon primary site in the chest and pulmonary metastases, treated successfully with surgery and vismodegib. A 51-year-old woman presented with a large pigmentary lesion of the chest, close to the sternum. She had the lesion for $>25$ years and lately noticed multiple facial lesions. The diagnosis of multiple BCC was suspected and a punch biopsy of the primary lesion was performed. Diagnosis was confirmed by immunohistochemistry (BerEp4+, EMA- phenotype). After excision, staging with a thorax computed tomography scan revealed metastatic micro-nodules in the left lung, confirmed histologically after video-assisted thoracic surgical biopsy. Adjuvant chemotherapy with vismodegib was proposed and administered. At 30 days follow-up, thorax computed tomography scan was unaltered and her facial lesions showed significant regression. Although prognosis remains poor, early diagnosis and prompt management complimented by novel biological agents, like vismodegib, targeting disease pathogenesis, seems to bring promising results.

\section{Introduction}

BCC represents $80 \%$ of all nonmelanotic skin malignancies. It usually affects elderly Caucasian men and skin regions mostly exposed to the sun. ${ }^{1,2}$ About two-three million people/year get affected globally, 730-1000/100.000 in the United States, 1000-2000/100.000 in Australia and $132 / 100.000$ in Europe. ${ }^{1,3}$ BCC is a slowly growing, usually not metastatic due to its strong stromal dependence, ${ }^{4}$ but invasive cancer. Its size varies greatly, with giant $\mathrm{BCCs}$ of maximum diameter $>5 \mathrm{~cm}$ representing $1 \%$ of all lesions, while multiple lesions are often associated with hereditary disease like in Gorlin, Rombo or Bazex syndromes. ${ }^{5}$

Metastatic BCC (mBCC) incidence ranges between $0.003 \%$ and $0.55 \%,{ }^{1,2,6}$ with a men/women ratio between $2: 1$ and $3: 1{ }^{7}$ and head, neck and face regions get predominantly affected. ${ }^{1,2}$ It spreads primarily to the lymph nodes, followed by the lungs, bones and other organs. ${ }^{1,2,6}$ Only $\sim 400$ cases have been reported since 1894. ${ }^{2,7,8}$ Therefore, the present case, a multiple, non-syndromic $\mathrm{mBCC}$ of the chest, treated successfully with surgery and vismodegib (GDC-0449, Erivedge ${ }^{\mathbb{}} /$ Roche Registration Ltd, Germany) at a tertiary Greek hospital, is rather rare and worth mentoning.

\section{Case Report}

A 51-year-old female with a history of depression, presented with a large pigmentary lesion close to the sternum (Figure 1a), 6×3 centimeters in dimension, with dermoscopic features of large converging blue-black ovoid nests, central ulceration and peripheral regression (Figure 1b). She had the lesion for more than 25 years, while multiple facial plaques and papules (Figure 1c), also pigmentary, with blue-gray and blue-black ovoid nests and ulcerations, as seen dermoscopically, and many with a distinct pearl-like edge, appeared during the last three years. No lymphadenopathy was present. Multiple BCC was suspected and a punch biopsy was performed. Pathology results showed a neoplasm with morphological characteristics of either a skin adnexal
Correspondence:Konstantinos Roditis, Zinonos 32, Halandri, Attica, 15234, Greece. Tel.: +30.697.6686951.

E-mail: roditis.k@gmail.com

Key words: Basal cell carcinoma, non-syndromic, skin cancer, lung metastases, vismodegib.

Acknowledgements: The authors would like to thank Dr. P. Maniatis, consultant radiologist, head, depart-ment of computed-assisted tomography (CT) and interventional radiology at Konstantopouleio Neas Ionias-Patission General Hospital, Nea Ionia, Athens, Greece, for CT scanning of the patient during staging and fol-low-up.

Contributions: K.R., G.M., O.N. and K.L. conceived the study. G.M. performed the biopsies. G.M., K.L. and O.N. were responsible for the patient's clinical care, treatment and follow-up. K.P. and A.K. performed histopathology and imaging. K.R., K.L., K.P. and A.K. were in charge of overall direction and planning. K.R. and K.L. wrote and revised the manuscript with input from all authors. All authors approved the final version of the manuscript.

Conflict of interest: The authors declare no conflict of interest.

Funding: No funding was received.

Please cite this article as: Roditis K, Metaxas $G$, Neofotistou $O$, et al. Multiple non-syndromic basal cell car-cinoma with the chest as primary site and lung metastases: a rare case. Dermatol Rep 2021;13:9106.

Received for publication: 15 February 2021. Revision received: 20 April 2021.

Accepted for publication: 21 April 2021.

This work is licensed under a Creative Commons Attribution-NonCommercial 4.0 International License (CC BY-NC 4.0).

${ }^{\circ}$ Copyright: the Author(s), 2021

Licensee PAGEPress, Italy

Dermatology Reports 2021; 13:9106

doi:10.4081/dr.2021.9106

tumor or a BCC. More specifically, it was characterized as a basaloid epithelial tumor of the epidermis with peripheral palisades, with mitotic crowded centrally located nuclei, presence of various pale cells and few infiltrating strands of atypical epithelium. Histochemistry and immunohistochemistry uncovered a BerEp4+, EMA - phenotype (Figure 2), in favor of BCC, and surgical excision of the lesion was recommended. Final pathology validated $\mathrm{BCC}$ diagnosis, revealing a tumor with microscopic characteristics of $\mathrm{BCC}$, 
with various sites of focal ulceration, partial infiltration of the subcutaneous nerves and vessels, and minor focal metaplasia to metatypical basal cell carcinoma, with negative excision margins. A thorax computer-assisted tomography scan (CT) for staging revealed a 7-mm nodule (Figure 3a) and several "ground glass"-like micronodules in the left lung, representing possible distant metastases. A videoassisted thoracic surgical (VATS) biopsy of the 7-mm nodule was performed followed by histological examination of the specimen, revealing nests of basaloid epithelial cells with peripheral palisading, thus confirming its metastatic nature. Due to disease extent, adjuvant treatment with vismodegib was initiated at $150 \mathrm{mg} /$ day and she was discharged. An unaltered CT-thorax (Figure $3 b$ ) and regression of her facial lesions were observed at 30-days follow-up.

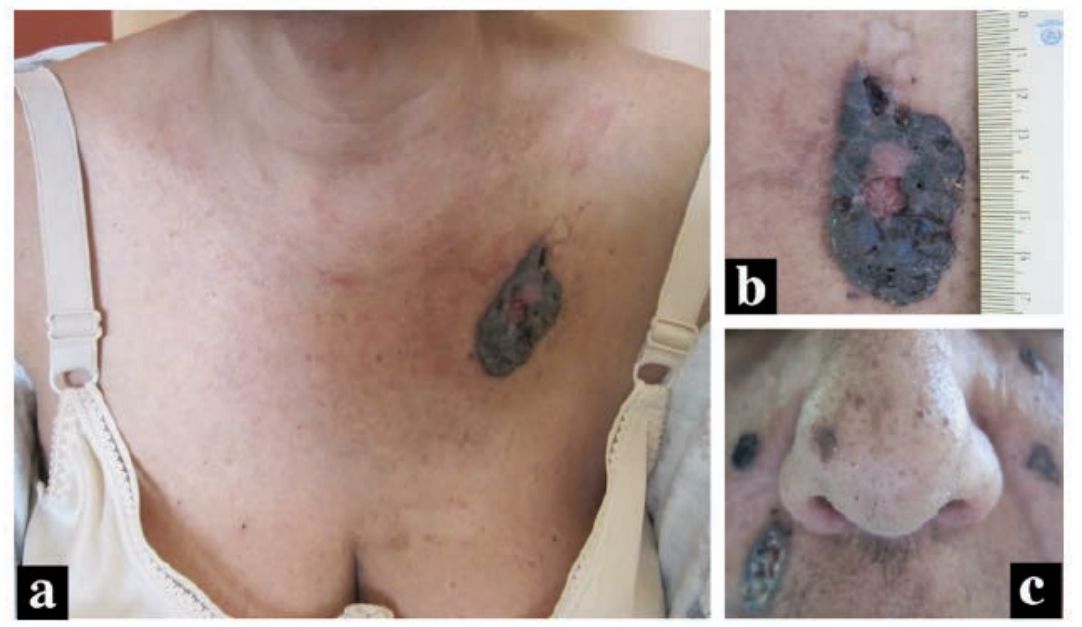

Figure 1. a) Unusual location of the primary lesion in the chest, close to the sternum, b) close view $(5 \times)$ of the primary lesion, sized $6 \times 3 \mathrm{~cm}$ with central ulceration and peripheral regression, c) multiple facial pearl-like edged lesions with partial ulceration.

\section{Discussion}

The usual BCC case is a male, middleaged patient, with a singular small $(<5 \mathrm{~cm})$ non-metastatic lesion located in the head or neck. Here, we present a multiple $\mathrm{mBCC}$ in a female patient, with a giant $(6 \times 3 \mathrm{~cm})$ primary lesion located in the chest and left lung metastases, in other words, an unusual and aggressive form of this common disease.

Known risk factors, namely light skin color (Fitzpatrick skin types I and II), smoking history and occasional exposure to ultraviolet-A light (UVA), were present, while exposure to arsenic, ionizing radiation or dry ice, immunosupression, albinism, keratoacanthoma or xeroderma pigmentosum were absent. Hereditary multiple BCC was excluded in our case due to negative family history of genodermatoses. ${ }^{1,3,5}$

Although the fourth, fifth and sixth decades are considered the mean or median age of presentation of the primary lesion, ${ }^{2,7}$ our patient developed her first BCC at age 26. Nevertheless, it was left untreated for 25 years and not only it metastasized, but she also developed multiple primary lesions, as expected by described high 1-year and 5year cumulative risks to develop secondary lesions. ${ }^{5,6,9}$

Metastatic BCC occurs two times more often in males than females with the most common site of metastases being lymph nodes, followed by the lungs. Presentation age, the site and size of primary lesion, perineural and perivascular spread, duration and recurrence of disease, incomplete resection or positive margins, multiple
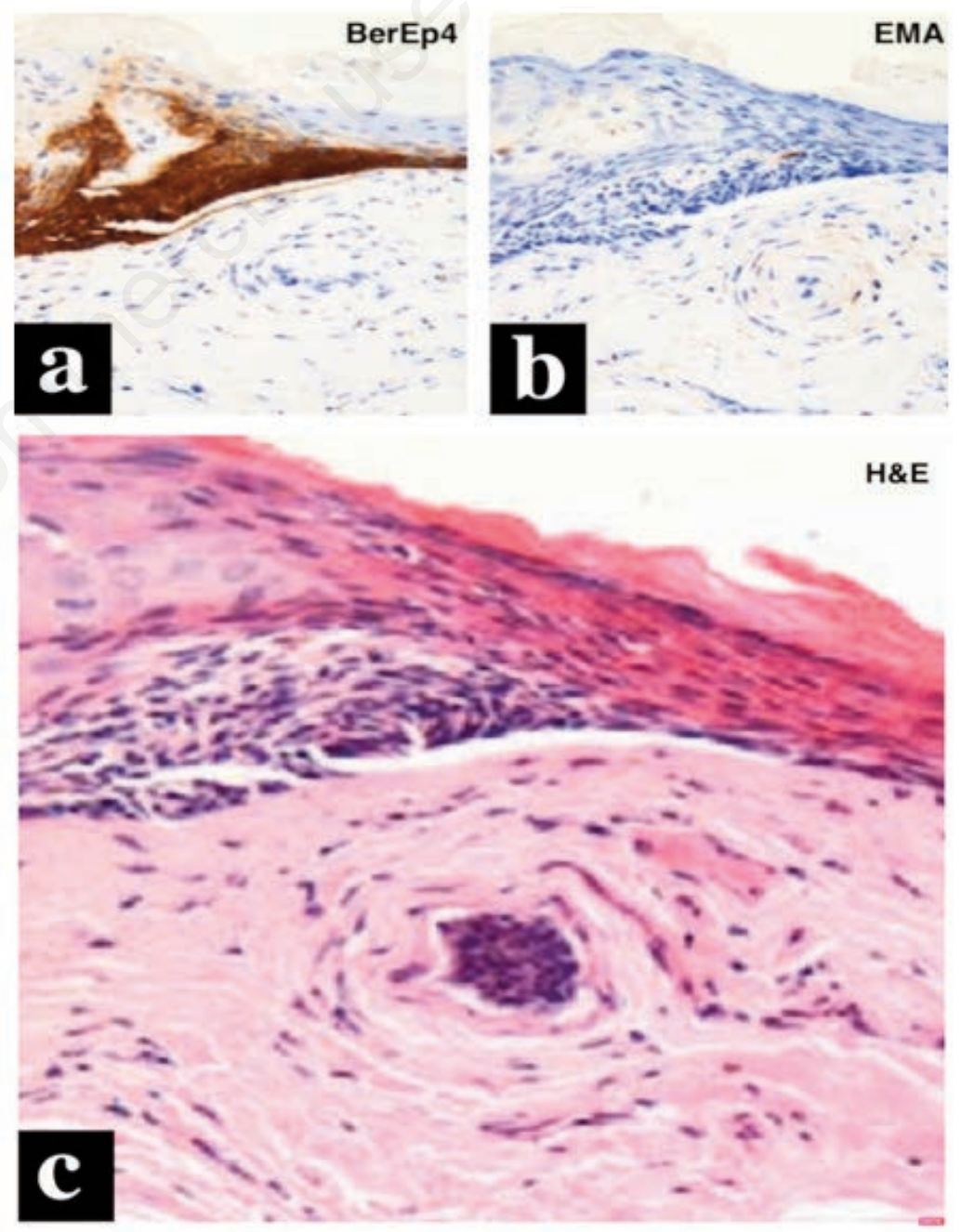

Figure 2. Comparison BerEp4(+) immunohistochemistry stain (200x) (a) with EMA(-) $(200 \times)(b)$ and $H \& E(400 \times)$ (c) stains of the primary tumor punch biopsy. 


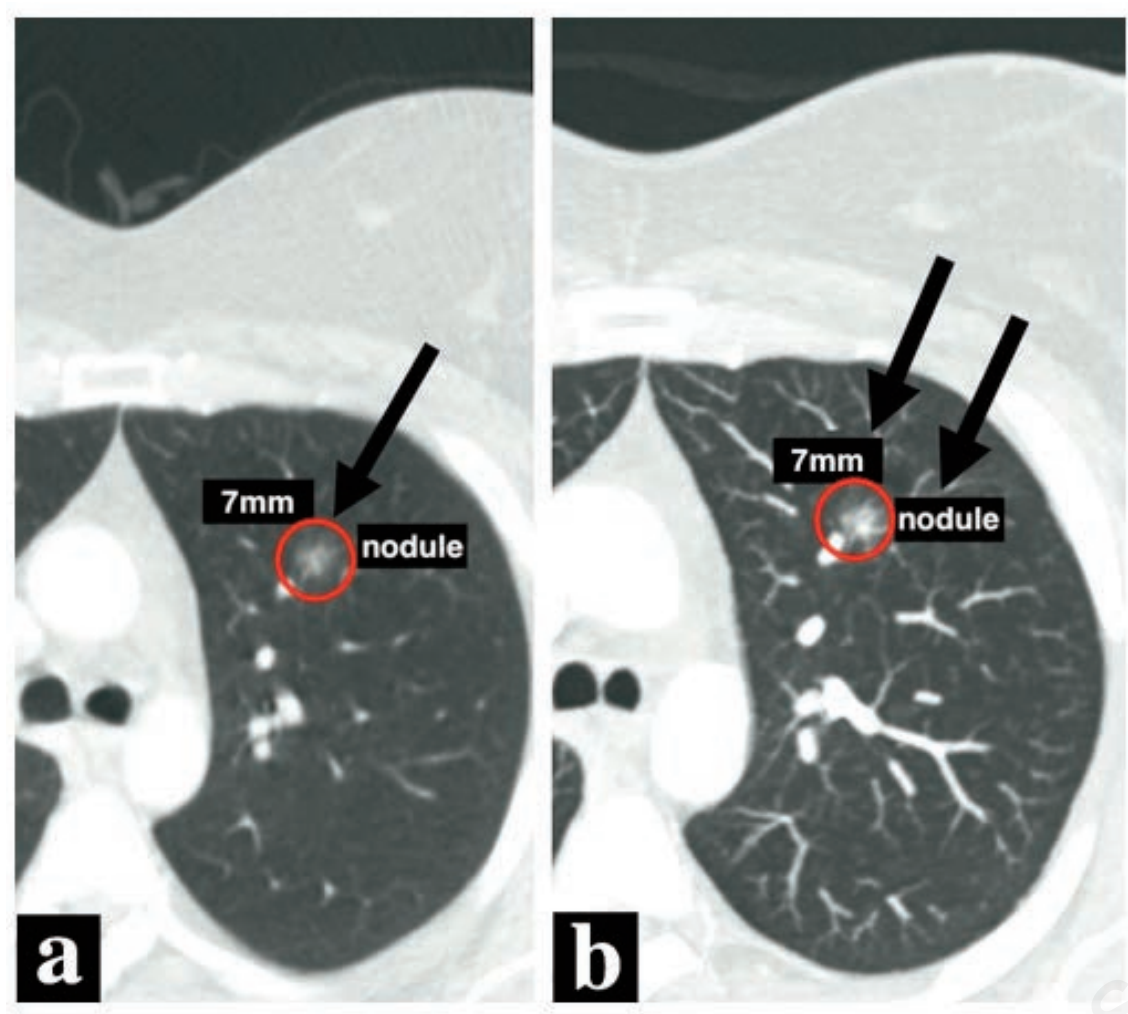

Figure 3. CT-thorax scan images of the metastatic $7 \mathrm{~mm}$ left lung nodule: (a) single arrow - at presentation, (b) double arrow - at 30-days follow-up.

tumors and aggressive histological types may predict metastasis. ${ }^{1-3}$ Tumors greater than $3 \mathrm{~cm}$ in diameter have a $2 \%$ incidence of metastasis and/or death. , $^{2,6}$ Prognosis is poor, ${ }^{1,2,6,7}$ with a longer average survival of three-and-a-half to seven years, when lymphatic spread is involved, ${ }^{2}$ in contrast with an average of eight months to two years that accompanies hematogenous metastases. ${ }^{1,2,4,7}$

Surgery and radiotherapy combined or not with targeted therapy are the most common therapeutic options used. ${ }^{2,5}$ Aberrant activation of the Hh pathway has been associated with pathogenesis of sporadic, non-syndromic BCCs as well as in hereditary syndromes with multiple BCCs. ${ }^{1,5}$ Small molecule antagonists of the Hedgehog pathway, like vismodegib and sonidegib, can both be used as adjuvant targeted therapy in locally advanced disease, however, only vismodegib is indicated for metastatic disease. ${ }^{10}$ Although nausea/loss of appetite and vomiting, diarrhea or constipation, weight and hair loss, muscle spasms and joint pain have been described among others as common side-effects, response rates can reach $37 \%$, thus proving their therapeutic potential against mBCC. ${ }^{2}$ Our patient received this novel therapy and showed positive primary results.

\section{Conclusions}

In conclusion, the earlier the identification of this potentially curable form of cancer, the better the therapeutic results and the least the morbidity complications. Comprehensive sensitization of the population and awareness raising by all relevant physicians, as well as fostering a healthy doctor-patient relationship regarding communication of knowledge on the primary $\mathrm{BCC}$ disease and its metastatic potential, can indeed play a crucial role in increasing favorable outcomes. Precise excision of the primary tumor and adjuvant targeted therapy with Hedgehog inhibitors, can bring promising results in aggressive cases of $\mathrm{mBCC}$ and must be encouraged.

\section{References}

1. Rubin AI, Chen EH, Ratner D. Basalcell carcinoma. N Engl J Med 2005;353: 2262-9.

2. Piva de Freitas P, Senna CG, Tabai M, et al. Metastatic basal cell carcinoma: a rare manifestation of a common disease. Case Rep Med 2017;2017: 8929745.

3. Wu S, Han J, Li WQ, et al. Basal-cell carcinoma incidence and associated risk factors in U.S. women and men. Am J Epidemiol 2013;178:890-7.

4. Robinson JK. Risk of developing another basal cell carcinoma: a 5-year prospective study. Cancer 1987;60:11820.

5. Kim DH, Ko HS, Jun YJ. Nonsyndromic Multiple Basal Cell Carcinomas. Arch Craniofac Surg 2017;18:191-6.

6. Robinson JK, Dahiya M. Basal cell carcinoma with pulmonary and lymph node metastasis causing death. Arch Dermatol 2003;139:643-8.

7. Wysong A, Aasi SZ, Tang JY. Update on metastatic basal cell carcinoma: a summary of published cases from 1981 through 2011. JAMA Dermatol 2013;149:615-6.

8. Lattes R, Kessler RW. Metastasizing basal-cell epithelioma of the skin; report of two cases. Cancer 1951;4:86678.

9. Marcil I, Stern RS. Risk of developing a subsequent nonmelanoma skin cancer in patients with a history of nonmelanoma skin cancer: a critical review of the literature and meta-analysis. Arch Dermatol 2000;136:1524-30.

10. Meiss F, Andrlová H, Zeiser R. Vismodegib. Recent Results Cancer Res 2018;211:125-39. 